\title{
AN INSTRUCTOR'S EXPERIENCE OF IMPLEMENTING BLENDED LEARNING IN ENGINEERING: BENEFITS AND CHALLENGES
}

\author{
Samer Adeeb, ${ }^{1}$ Carol Brown, ${ }^{2}$ and Norma Nocente ${ }^{2}$ \\ ${ }^{1}$ Department of Civil and Environmental Engineering, Faculty of Engineering \\ ${ }^{2}$ Centre for Teaching and Learning \\ University of Alberta \\ adeeb@ualberta.ca; carol2@ualberta.ca; nnocente@ualberta.ca
}

\begin{abstract}
This paper discusses how a blended learning approach was implemented in two courses in the Civil and Environmental Engineering program at the University of Alberta: CivE 398 (Introduction to Continuum Mechanics); and CivE 295 (Civil Engineering Analysis II). During the blend, two approaches were used to convert content to an online format: first was the development of online videos that were used to complement either in-class or laboratory activities. The second approach was the development of an online textbook (https://sameradeeb.srv.ualberta.ca) with interactive examples which replaced a traditional paper textbook. For each approach, the tools that were used along with the associated costs will be presented. Changes to in-class or lab activities will be also be discussed, along with the associated challenges, and perceived advantages of the blended approach, from the instructor perspective. In addition, the preliminary results of student surveys related to one course (CivE 398) are briefly summarized.
\end{abstract}

Keywords: blended learning, flipped learning, online textbook, instructor perspective

\section{INTRODUCTION}

Blended, or 'flipped' learning is an instructional approach which involves the introduction of class content through videos, readings, or other media on students' own time (i.e. as homework). Under this approach, class time which was traditionally used for lecture is now available for more active and engaging learning experiences such as problem solving, group work, research, or other applications. Advocates of blended learning indicate that it offers an opportunity to enhance the creativity and innovation in engineering programs [11] and can encourage students to develop the independent learning skills required to be practicing engineers [8]. Others have noted that flipped courses may offer more flexibility in scheduling for both students and instructors [4, 7].
Further, there is some evidence that, when compared to traditional lecture courses, flipped classes have the potential to improve students' understanding of engineering concepts, and at worst 'do no harm' $[8,9]$.

While there is a growing body of literature on blended learning at the postsecondary level, a need for more studies on the nature and effectiveness of blended models has been identified [7], particularly within the engineering context [10]. Related to the nature of blended courses, in particular, there are a number of papers that detail how engineering courses have been adapted to a blended format [see 1, 8, 10,12], though fewer papers describe the instructor experience during this transition [see 2, 5]. Further, while there is some literature to address the development of online textbooks in engineering [6], we located no papers that discuss online textbook development and implementation within a blended engineering context. As such, this paper seeks to add to this body of work through a description of how two courses, CivE 398 (Introduction to Continuum Mechanics), and CivE 295 (Civil Engineering Analysis II) were converted to a blended format through the development of online videos and an online, interactive textbook. In addition, this paper describes the instructor's experience of this process of development and implementation, including perceived benefits and challenges. While the results reported here are not generalizable across broad contexts in engineering education, they do provide significant insight into the practical considerations surrounding implementation of blended approaches within engineering courses. Further, they may help to guide instructors, departments, and institutions in the planning and development of subsequent blended courses within engineering contexts.

\section{APPROACH TO BLENDING}

\subsection{Description of Courses Before the Blend}

Two courses within the Civil and Environmental Engineering program at the University of Alberta (taught 
by the lead author) were selected for implementation of the blended approach: CivE 398 (Introduction to Continuum Mechanics); a third-year course covering advanced solid mechanics, and CivE 295 (Civil Engineering Analysis II); a second-year course covering an introduction to numerical analysis. Prior to implementation of the blended approach, both courses were taught using a traditional, lecture based approach with students undertaking weekly readings and assignment work on their own time, outside of class.

On average, CivE 398 is attended by 150 students in the Fall term in both the traditional and co-op stream of Civil Engineering. The students attend two 1.5 hour lectures per week. Each class is divided into two groups; each group attending a 1 hour seminar per week. The lectures were used to deliver content and demonstrate examples while the seminars were used to discuss the assignments with students. Students in CivE 398 relied on printed course notes and lecture notes provided by the instructor. For the period Fall 2011 up to and including Fall 2014, a traditional (paper) textbook created by the instructor was used.

CivE 295 is typically attended by approximately 190 students in the Winter term in all streams of Civil Engineering and Environmental Engineering. The students attend three 1 hour lectures per week. CivE 295 students are also divided into 6 groups; with each group attending a 2 hour computer lab per week. The lectures were used to deliver content while the labs were used to implement various numerical techniques in Matlab. A traditional textbook on numerical analysis was the course reference, in addition to printed course notes provided by the instructor.

\subsection{Blended Course Development}

Initial planning and direction of blended course development was initiated by the lead author (instructor of the course); an educational developer helped support the instructor and liaise between the instructor and others (such as technical support persons; copyright office, etc). One motivating factor driving the initial design was the instructor's desire to increase the accessibility of course content (i.e. increase the online availability of course materials) for students who miss lecture or need additional review. Second, the instructor was becoming frustrated with the process of revising the current course textbook, which had been developed by the instructor. Even simple revisions (such as the correction of typos) required correspondence with the book editor and were not able to be undertaken expediently. In addition, any changes made would not be reflected in the book until a subsequent version was printed. With these factors in mind, three areas of change were initiated in each blended course: online videos were created, the textbook was adapted to an online format, and changes to face-to-face interactions with students were also undertaken. Each of these areas will be discussed in the following sections.

2.2.1. Creation of Online Videos. A key element of the blended approach for both courses was the creation of online videos. For CivE 398, these videos were intended to replace the face-to-face lecture content across a number of topics, freeing up time in lecture periods for additional examples, answering student questions, or extension of material. The process of development of these videos was as follows: The course content was divided into topics such that each topic would be covered in a 5 to 15 minute online lecture. For each topic, a Microsoft PowerPoint presentation was first created which was then transferred into Explain Everything ${ }^{\mathrm{TM}}$. The instructor then narrated the presentation, recording audio in Explain Everything, and then the lecture was exported as a video file. The production team in the Centre for Teaching and Learning at the University of Alberta then provided proper compression to the videos and edited them for clarity. Once complete, videos were uploaded to a class YouTube channel and then students could access the videos via links through the course management system (eClass Moodle). In total, 37 videos were created and can be found on the YouTube play list: https://www.youtube.com/watch?v=K4QpWsWFtM4\&lis $\mathrm{t}=$ PLWlJvChadvVz0kK6qf_W6YI3qWBydZtPL. The approximate time investment for the development of the videos was as follows: approximately 280 hours by the instructor, 183.25 hours by the production team, and 45 hours by the educational developer. The approximate cost to develop the videos was $\$ 4581.25$ in production team support and $\$ 2250.00$ for the educational developer. This work was completed over approximately four months during the summer of 2015. Videos were labelled by topic and numbered in a way that they corresponded to online textbook material for ease of student reference. Students were typically assigned to watch 2 to 3 videos for each 'lecture' period.

For CivE 295, online videos were created not to replace lecture content, but rather to serve as lab tutorials in order to facilitate students' efficiency and understanding of material within the weekly lab sessions, and reduce the strain on the instructor during the laboratory periods. In particular, the online tutorials aimed at teaching the students how to use the chosen software (Mathematica) to implement various numerical analysis algorithms. For each week's lab, the online tutorials typically involved introductory tutorials to present the various Mathematica keywords needed for the lab, and a tutorial on how the particular lab could be implemented efficiently in the Mathematica environment. 
The development of these videos included the hiring of an undergraduate assistant (UA) who had previously completed the course to create screen-cast tutorials within the software Mathematica and employing Microsoft Excel. The process of development of these videos was as follows: a schedule for completion was negotiated between the instructor, educational developer and UA. The instructor provided the UA with guidelines for the lab content required in each video (and supporting documentation such as lab descriptions from previous years and the online course textbook). The UA then drafted a script for each tutorial video prior to filming; scripts were reviewed by the instructor for content accuracy and educational developer for flow, clarity, and organization. Feedback was provided to the UA; the UA then recorded the videos in a screencast style using a Wacom tablet and stylus with the software Epic Pen, and Camtasia Studio 8. Draft videos were then reviewed by the instructor and educational developer; any necessary revisions were undertaken by the UA or, when needed (such as occasional text layovers to emphasize points or indicate corrections) by the production team at the Centre for Teaching and Learning. Videos were output in MP4 format and uploaded to a course YouTube channel which students could access through their course (eClass Moodle) page. Students were expected to watch these videos prior to attending their labs, but also had access to these videos during their lab sessions (via their own personal electronic devices). In total, 29 videos (ranging from 5 to 15 minutes in length) were completed over a four month period. The total number of hours spent on video creation was approximately: 40 hours by the course instructor, 30 hours by the educational developer. The UA was hired for four months (35 hours/week) during the summer of 2016 for the task of creating these videos. The approximate cost associated with production was: $\$ 6,000.00$ for UA; $\$ 1,500.00$ for educational developer, and $\$ 2,000.00$ for a Microsoft Surface Pro 3 Tablet.

2.2.2. Creation of Online Textbook. When undertaking the creation of the online textbook, the first step was to define its design criteria. The first criterion was the ease of editing; in particular, section numbering needed to be automatic such that if a new page or topic was inserted, the following pages or sections would be renumbered. Another important criterion was that the website should be visually pleasing with an organized table of contents. It was also important to the instructor that comments were permitted on the site so that readers could help in the development process by identifying pages or sections that have typos, errors, omissions, or lacked clarity. The instructor also required that each section have a shareable hyperlink so that content could easily be shared between the instructor and the students when it was necessary to answer specific questions or to direct students to specific topics or examples. Due to the nature of the material, it was important to be able to clearly display mathematical expressions. Finally, the website needed to be able to include "interactive examples" where the input could be changed by the site visitor and would instantaneously display the new answer.

While a number of available packages that could partially address these design criteria, the instructor opted to employ WordPress and a custom designed theme hosted on a virtual server by Information Services and Technology (IST) at the University of Alberta. An external web designer was hired and modified one of the available WordPress themes to allow the pages to be automatically numbered according to their hierarchy. Inside each page, the first two subheadings would be automatically numbered according to the page number within the hierarchy. Additionally, a "page contents" menu was made to appear inside each page with the first two subheadings (For example see: http://sameradeeb.srv.ualberta.ca/variationalprinciples/the-principle-of-virtual-work/). In addition, each subheading was automatically given a hyperlink (For example: http://sameradeeb.srv.ualberta.ca/variationalprinciples/the-principle-of-virtual-work/\#problems-22). In addition, as WordPress allows various developer plugins, the free "WP QuickLatex" plugin was installed which allows the use of inline formulas and displayed equations using native Latex syntax. Finally, commenting sections were automatically built-in at the bottom of each page (For example:

http://sameradeeb.srv.ualberta.ca/variational-

principles/the-principle-of-virtual-

work/\#page comments). The option of "moderating each comment before posting" was also selected.

One of the important features of the online textbook, particularly in comparison to a traditional paper textbook, is the availability of interactive examples where the site visitor can input or change numbers and see the effect on the output. For example see: http://sameradeeb.srv.ualberta.ca/linear-algebra/linearvector-spaces/basic-definitions/\#basis-and-dimensions4. Alternatively, by using a slider, the student can visualize the effect of how a particular value changes the outcome. For example see: http://sameradeeb.srv.ualberta.ca/introduction-tonumerical-analysis/numerical-integration/\#rectanglemethod10. For these tools, it was decided that the most convenient method would be to utilize a server based software where the computations are completed on the server side and the output is displayed on the client (site visitor) computer. In particular, the tool that was selected for developing these examples was webMathematica. webMathematica is a set of commands written using Java installed on the server side, which can evaluate regular Mathematica code and convert it into a graphic object that can be embedded in the page. Given that the textbook developer was already familiar with the Mathematica 
software, and webMathematica requires no knowledge of the Java language (only basic HTML knowledge), webMathematica was the package selected to create the interactive examples. The package was installed on a Linux server by first installing Mathematica, then, a a package was installed that allowed the implementation of Java code (Apache Tomcat). Next, webMathematica was installed on Apache Tomcat as per the instructions provided by Mathematica. Finally, the content of each interactive example was created in the Mathematica software (which allows dynamic computations) and then converted into a java script file (*.jsp) using the webMathematica keywords and then embedded inside the page using an "iframe".

The costs associated with the website development is as follows: The web designer initial setup cost $\$ 5,000.00$, the annual hosting of the website on the University of Alberta servers cost just under \$2,000.00, and the Mathematica academic license each year cost approximately $\$ 350.00$. It is worth noting that webMathematica is free for academic users of the software provided they purchase a yearly academic license of Mathematica. Also, hosting on the University of Alberta servers includes hosting, backup, and some limited system administration. While using a third party hosting can be cost effective, the instructor opted to use IST to maintain the link with the university.

The actual content of the website was in development since 2012. In particular, initially, the website was developed by converting the lecture notes of CivE 398 into the website format. Following that, some advanced material, taught in graduate courses, was added in 2013 and 2014. The chapter on finite element analysis was added in the summer of 2015. Finally, the contents of CivE 295 were added in 2015. It is difficult to quantify the time spent developing and adding material to the website as the instructor did not keep a strict record. In addition, while the materials in some sections were straightforward to add to the site, they took considerable time to research and lay out in concise and complete manner. Developing such an online textbook is a huge undertaking that can be even more daunting that creating a paper based textbook. However, the instructor noted several advantages: having the contents available online is means that all interested individuals are enabled to be editors. In addition, correcting typos, adding sections and examples, or clarifying concepts is instantaneously applied to the text in an online text (rather than requiring reprinting). Finally, the online interactive examples can assist to engage readers and also serve as illustrative tools during the actual lecture time.

2.2.3. In-Class / Lab Activities. While the initial focus of course development was on creation of online resources, the implementation of the blended approach also impacted face-to-face components of the CivE 398 and 295 courses in a number of ways. In particular, the creation of the online videos and the online textbook for CivE 398 enabled more efficient use of the face to face lecture time. While lectures were still prepared using PowerPoint slides similar to those in the online videos, additional slides were added at the end of each topic with a focus on one of three extension areas: real world examples, activities, and interactive examples. Real world examples typically included some pictures or videos illustrating the applications of the topic in the engineering world. For example, when discussing the failure of materials, Youtube videos of steel and/or concrete materials failing were shown and discussed during the lectures. The second extension was to introduce class activities where the students were asked some problems and then required to work together to think about the possible answers to these problems. After a short period of time to work on the problems, the instructor would go through the solutions in class. The third type of extension activity was based on the interactive examples in the online textbook to illustrate the effect of changing some variables on the output. As an example, the tool shown in the section: http://sameradeeb.srv.ualberta.ca/linearalgebra/linear-vector-spaces/basic-definitions/ was used in class to show how a vector $v=(3,4)$ can be written in terms of any two linearly independent vectors, which in turn can be used as a basis for a two dimensional space.

For CivE 295, the online textbook with interactive examples are also used in class to visually illustrate concepts to students and encourage discussion. As an example, the tool in the rectangle method section in: http://sameradeeb.srv.ualberta.ca/introduction-tonumerical-analysis/numerical-integration/\#rectanglemethod10 allows the student to see how the method can be implemented in three different ways to calculate the area underneath the curve. The effect of increasing the number of rectangles on the accuracy of the numerical results can be visualized using the tool.

For the labs of CivE 295, the existence of the videos changed how the labs were conducted. In the first year of the instructor teaching this class (non-blended format), the first twenty minutes of each lab were used to explain features of the Mathematica software, discuss the numerical algorithm relevant to that particular lab, and identify how the software can be used to implement the particular algorithm. In the second year of the course, after the online tutorials were created, the lab time was used more efficiently because students came in to the lab prepared for the topic (having watched the videos beforehand) and had very specific questions regarding their implementation of the numerical algorithm.

\section{BENEFITS AND CHALLENGES (FROM THE INSTRUCTOR PERSPECTIVE)}


From the instructor perspective, there were a number of benefits and challenges related to implementing the blended approach in CivE 398 and 295.

For CivE 398, two major advantages of implementing the blended approach were noticed by the instructor. The first advantage was that the online videos freed up more time during lecture to discuss the applications of the material studied. The instructor felt this was particularly important given that solid mechanics can sometimes be perceived as 'dry' by students, and is often taught from a theoretical perspective. The second major advantage, based on anecdotal feedback and discussions with students, was that while students found the material to be difficult to understand at first, having the online videos available to re-watch and review at their own pace helped them better understand the content.

For CivE 295, two major advantages of creating the lab tutorials were realized. The first advantage was freeing the instructor's time. As the class is typically divided into 6 lab groups, the instructor used to (prior to the blended approach) give a twenty minute introduction to the lab for each group. Under the blended model, these introductions were recorded for students to view, along with other tutorials, prior to entering the lab. As such, under the blended model, the instructor was now free during the lab to move around the space, address the students' questions, and offer feedback and support. This allowed the instructor to build a stronger rapport with the students and thus made lab periods more enjoyable for the instructor. The second major advantage noted by the instructor was the comfort and proficiency of students in the lab under the new approach. In particular, the instructor noticed that in the first year, when the online tutorials were not available, the students became frustrated with learning the new software along with implementing the numerical algorithm. While sample code was available online:

http://sameradeeb.srv.ualberta.ca/introduction-to-

numerical-analysis/labs/ the students struggled to find the appropriate keywords to use. In the second year, after the implementation of the videos, labs ran more smoothly; the majority of the students were able to begin work immediately and implement the numerical algorithm with very few initial questions. While this evidence is anecdotal, the results of student surveys (forthcoming) should provide additional evidence as to students' experience in the lab.

Despite these advantages, there were some challenges related to implementation of the blended approach. One major challenge included the time-intensive process of adapting the course to a blended format (in particular, video and online textbook creation), which has been noted elsewhere $[3,8,10]$. This challenge was mitigated somewhat by the support available through the University's Centre for Teaching and Learning, made accessible by grant money provided by the institution
(Provost Digital Learning Committee Blended Learning Award), and through the hiring of an undergraduate student assistant (CivE 295), however a considerable time investment on the part of the instructor was still required. Other challenges to implementation include the initial costs associated with development of the website and videos (outlined in a previous section) as well as the annual costs associated with the hosting of the online textbook.

In addition to the initial time investment involved, another challenge is the time and effort needed to ensure the online components of the blended course (textbook content and videos) are in working order and accessible to students, year after year. For example, the software webMathematica requires an annual upgrade. While only basic knowledge of Linux command line keywords is needed, the instructor finds it challenging to ensure, every year, that all the components are running smoothly. In addition, technical issues can become a major disadvantage when course content is primarily accessed online. For example, over the two years since the online textbook went live, the website server was down four times. One of these times was the evening before a midterm; the instructor had to call the university information technology emergency hotline to ask them to restart the server. Despite the investment of time needed to keep online course components running smoothly, the instructor has noted that the amount of preparation time for each blended course (after the first iteration of the course) has been an approximately equivalent to a nonblended course; time is just allocated to different tasks.

Another disadvantage for the instructor was that for CivE 398, the transition to more active learning approaches was more challenging than expected. In particular, the instructor has found it difficult to initiate class discussions and small group activities during the face-to-face lectures. For example, during the lecture time dedicated to activities, the instructor often asked the students to discuss a particular problem among themselves for a few minutes. This was sometimes met with silence or limited discussion, which the instructor found very awkward. This feeling of discomfort and uncertainty on the part of the instructor and tentative participation by students may be related to a number of factors: the (relative) inexperience of the instructor with implementing active learning approaches, the nature of students and the class (a large lecture with approximately 150 students; students may be 'used' to lecture style classes; classes are early in the morning, scheduled at 8:00am on Tuesdays and Thursdays), and the theoretical nature of the course (continuum mechanics). While not insurmountable, it is clear that in future iterations of the course, additional work and time will need to be spent by the instructor to focus on designing and implementing more engaging activities that initiate class discussions. It is the instructor's hope that, over time, enjoyment of 
teaching using the blended approach will increase, as it has in the lab sections of CivE 295, and has been noted elsewhere [10].

\section{PRELIMINARY STUDENT FEEDBACK}

While data is still being collected and analyzed related to the implementation of these blended courses (and will be described in subsequent publications), preliminary feedback from students is positive. Data from a voluntary, anonymous student survey $(n=42$ respondents out of 164 students enrolled) following the Fall 2016 CivE 398 course highlights several trends. In particular, students indicated a preference for the blended format, with $76.2 \%$ of students indicating that if the same course was offered in a variety of formats, they would prefer a blended online and face-to-face format over a completely online $(4.8 \%)$ or completely face-to-face $(19 \%)$ format. In addition, students had positive feedback regarding the online textbook: they found it useful $(85.5 \%$ agree or strongly agree), well organized and easy to navigate (76.2\% agree or strongly agree), and approximately half $(54.8 \%)$ of the students indicated that the interactive features within the online textbook as helped them better understand the content.

Overall, students in the Fall 2016 CivE 398 course found the online tutorial videos (lecture videos) helpful (64.3\% agree or strongly agree), however students did indicate that if given the choice between attending lectures face-to-face or accessing lectures online, $76.2 \%$ of students would prefer face to face lectures. Student comments highlighted that some students preferred the online lectures, because they could watch them at a time convenient for them, and re-watch them if needed for purposes of review. Other students indicated they did not choose to watch the online lectures because they felt the face-to-face lectures were more than sufficient. Further analysis of data (including student interviews) from both CivE 398 and 295 will provide greater insight into the strengths and weaknesses of the blended approach, from the student perspective.

\section{CONCLUSIONS}

Based on experiences with two undergraduate engineering courses at the University of Alberta, we found the initial implementation of blended learning to be time consuming and costly, particularly when developing both video tutorials and online textbook materials to span the content of entire courses. In addition to these up-front challenges, maintaining quality online resources requires ongoing maintenance and attention on the part of the instructor. Transitioning to more active learning approaches in face to face contexts may also result in some 'growing pains' for both students and the instructor.
Despite these challenges, a number of benefits of the blended approach were also identified. In particular, class or lab time was freed up to allow for application of content, problem solving, and asking questions. In the CivE 295 blended course, in particular, the instructor had more time to address student questions, provide feedback, and discuss concepts with students than was previously possible. This made the laboratory sessions more enjoyable for the instructor. The instructor also perceived the students to be more confident and prepared in laboratory sessions, after implementation of the blended approach. Preliminary data from student surveys in CivE 398 also highlighted that students had positive perceptions of the blended course, and preferred the blended format over completely online or face-to-face formats, as has been noted elsewhere [10, 12]. Finally, the instructor noted that once the blended courses were developed, the time needed to prepare for subsequent offerings of the course was approximately equivalent to non-blended courses. Research related to these courses is ongoing; future work will seek to further investigate student perceptions of the blended approach, as well as impacts on student achievement and engagement.

\section{Acknowledgements}

The authors would like to acknowledge the support of funding granted by the Provost's Office at the University of Alberta, NSERC, and the Office of the Dean (Engineering).

\section{References}

[1] Konstantinos Apostolou, "Effectiveness of blended learning for an energy balance course," in Proc. CEEA Canadian Engineering Education Conf., CEEA16, (Halifax, NS; 19-22 June 2016), paper 085, 5 pp., 2016.

[2] Theodore Branoff, and Eric Wiebe, "Face-to-face, hybrid, or online?: Issues faculty face redesigning an introductory engineering graphics course," Engineering Design Graphics Journal, vol. 73, no. 1, pp. 25 - 31, 2009.

[3] Michael Derntl and Renate Motschnig-Pitrik, "The role of structure, patterns, and people in blended learning," The Internet and Higher Education, vol. 8, no.2, pp. 111-130, 2005 .

[4] Rebecca Francis and Susan J. Shannon, "Engaging with blended learning to improve students' learning outcomes," European Journal of Engineering Education, vol. 38, no.4, pp. 359-369, 2013. Available as of April 7, 2017 from http://login.ezproxy.library.ualberta.ca/login?url=http://searc h.proquest.com/docview/1418268207?accountid=14474

[5] Jeffrey Harris and Charles Park, "A case study on blended learning in engineering education," in Proc. CEEA 
Canadian Engineering Education Conf., CEEA16, (Halifax, NS; 19-22 June 2016), paper 132, 5 pp., 2016.

[6] Timothy R. Larson, "Developing a Participatory Textbook for the Internet," Journal of Engineering Education, vol. 90, no. 1, pp. 49-53, 2001.

[7] Betty Love, Angie Hodge, Neal Grandgenett and Andrew Swift, "Student learning and perceptions in a flipped linear algebra course," International Journal Of Mathematical Education In Science \& Technology, vol. 45, no. 3, pp. 317324, 2014. doi:10.1080/0020739X.2013.822582

[8] Gregory S. Mason, Teodora R. Shuman, and Kathleen E. Cook, "Comparing the effectiveness of an inverted classroom to a traditional classroom in an upper-division engineering course" IEEE Transactions on Education, vol. 56, no. 4, pp. 430-435, 2013. doi:http://dx.doi.org/10.1109/TE.2013.2249066

[9] Christopher Papadopoulos and Aidsa S. Roman, "Implementing an inverted classroom model in engineering statics: Initial results," in American Society for Engineering Education. American Society for Engineering Education, 27 pp., 2010.

[10] Lennart Svensson and Tom Adawi,,’Designing and evaluating a flipped signals and systems course," in Proceedings of the $14^{\text {th }}$ European conference on e-Learning - ECEL 2015 (Hatfield, UK 29-30 October 2015), 9 pp. 2015. Available as of April 7, 2017 from http://publications.lib.chalmers.se/records/fulltext/227895/lo cal_227895.pdf

[11] Evangelia Triantafyllou, Olga Timcenko and Lise B. Kofoed, "Student Behaviors and Perceptions in a Flipped Classroom: A case in undergraduate mathematics," in Proceedings of the Annual Conference of the European Society for Engineering Education 2015 (SEFI 2015) (Orleans, France 29 June - 2 July 2015), 8pp. 2015.

[12] Stephanie B. Velegol, Sarah E. Zappe and Emily Mahoney, "Successful flipped classes," ASSE Prism, vol. 24, no. 7, p. 41. Available as of April 7, 2017 from $\mathrm{http}: / /$ login.ezproxy.library.ualberta.ca/login?url=http://searc h.proquest.com/docview/1678629438?accountid=14474 